*For correspondence: d.fedosov@fz-juelich.de

\title{
Effect of malaria parasite shape on its alignment at erythrocyte membrane
}

${ }_{4}$ Anil K. Dasanna ${ }^{1}$, Sebastian Hillringhaus ${ }^{1}$, Gerhard Gompper ${ }^{1}$, Dmitry A. Fedosov ${ }^{1 *}$

$5{ }^{1}$ Theoretical Physics of Living Matter, Institute of Biological Information Processing and

6 Institute for Advanced Simulation, Forschungszentrum Jülich, 52425 Jülich, Germany

\begin{abstract}
During the blood stage of malaria pathogenesis, parasites invade healthy red blood cells (RBC) to multiply inside the host and evade the immune response. When attached to RBC, the parasite first has to align its apex with the membrane for a successful invasion. Since the parasite's apex sits at the pointed end of an oval (egg-like) shape with a large local curvature, apical alignment is in general an energetically un-favorable process. Previously, using coarse-grained mesoscopic simulations, we have shown that optimal alignment time is achieved due to RBC membrane deformation and the stochastic nature of bond-based interactions between the parasite and RBC membrane (Hillringhaus et alo, 2020). Here, we demonstrate that the parasite's shape has a prominent effect on the alignment process. The alignment times of spherical parasites for intermediate and large bond off-rates (or weak membrane-parasite interactions) are found to be close to those of an egg-like shape. However, for small bond off-rates (or strong adhesion and large membrane deformations), the alignment time for a spherical shape increases drastically. Parasite shapes with large aspect ratios such as oblate and long prolate ellipsoids are found to exhibit very long alignment times in comparison to the egg-like shape. At a stiffened RBC, spherical parasite aligns faster than any other investigated shapes. This study shows that the original egg-like shape performs not worse for parasite alignment than other considered shapes, but is more robust with respect to different adhesion interactions and RBC membrane rigidities.
\end{abstract}

\section{Introduction}

Malaria is a mosquito-borne infectious disease caused by a protozoan parasite of the genus Plasmodium. Prior to transmission, the parasite proceeds through both asymptomatic and symptomatic developmental stages in the host (Miller et al., 2002; Cowman et al., 2012; White et al., 2014). After an asymptomatic development stage within the liver, merozoites are released into the bloodstream. They have an egg-like shape with a typical size of approximately $1.5 \mu \mathrm{m}$ (Bannister et alo, 1986b; Dasgupta et al., 2014b). During the blood stage of infection, which is a clinically symptomatic stage, parasites invade healthy red blood cells (RBCs) and multiply inside them. This process aids parasites to evade the immune response. The total life-cycle within each infected RBC lasts for about $48 \mathrm{hrs,}$ after which the cell membrane is ruptured and new merozoites are released into the bloodstream.

Invasion of RBCs by parasites is a complex process that involves the following steps: $(i)$ initial random attachment, (ii) reorientation (or alignment) of the apex toward cell membrane, and (iii) formation of a tight junction followed by the final invasion (Koch and Baum, 2016; Cowman and Crabb, 2006). The parasite's apex contains the required machinery for the invasion process, and thus, apex alignment toward the cell membrane is a necessary step for a successful invasion 
to follow. Merozoite adhesion to a RBC is facilitated by proteins at the parasite surface which can bind to the cell membrane (Bannister et al., 1986b; Gilson et al., 2006; Beeson et al., 2016). Recent optical tweezers experiments provide an estimation for the force required to detach a parasite adhered to RBC membrane to be in the range of 10 to $40 \mathrm{pN}$ (Crick et al., 2014). Other experiments (Dvorak et al., 1975; Gilson and Crabb, 2009; Glushakova et al., 2005; Crick et al., 2013) demonstrate that the parasite is dynamic at the RBC membrane, and induces considerable membrane deformation during alignment. Furthermore, there is a positive correlation between such deformations and parasite alignment. The time required for the parasite to align is found to be on the order of $16 \mathrm{~s}$ (Weiss et al., 2015). Our recent investigation of the parasite alignment with adhesion modeled by a homogeneous interaction potential has confirmed the importance of membrane deformations for proper alignment, but the alignment times were found to be significantly less than $1 \mathrm{~s}$ (Hillringhaus et al., 2019). The main shortcoming of this model is that it produces only static membrane deformations and the parasite exhibits very little dynamics at the RBC surface. This model has been extended by including realistic bond-based adhesion interactions between the parasite and RBC membrane (Hillringhaus et al., 2020), which results in alignment times consistent with the experimental measurements.

A typical merozoite has an egg-like shape with the apical complex sitting on the pointed edge. Our previous work (Hillringhaus et al., 2020) suggests that parasite alignment occurs due to RBC deformability and stochastic fluctuations in bond dynamics. Stochastic fluctuations and consequent rolling-like (or rotational) motion of the parasite at the membrane surface are especially important at low adhesion strengths, as they facilitate alignment toward pointed apex. The egg-like shape naturally adheres to RBC membrane with its less curved side, as this adhesion state corresponds to the largest contact area. Then, a rotational motion of the parasite toward the apex is required to establish an apex-membrane contact. If parasite adhesion interactions with a membrane are strong, merozoite mobility is significantly suppressed, and the alignment is mainly facilitated through wrapping of the parasite by cell membrane, emphasizing the importance of RBC deformability. These are two major mechanisms for the alignment of an egg-like merozoite.

Even though most types of Plasmodium merozoites have an egg-like shape, the merozoite of Plasmodium yoelii changes its shape from an oval to a spherical shape right before its attachment followed by alignment and invasion (Yahata et al., 2012). The alignment time for Plasmodium yoelii is also reported to be longer than for Plasmodium falciparum (Yahata et al., 2012). It is not clear why the Plasmodium yoelii parasite adapts its shape before the alignment process at the RBC membrane. This raises a question whether the parasite shape has important advantages/disadvantages in the alignment process or simply results from the structural organization of its internal elements. Therefore, it is important to understand the effect of parasite shape in the alignment process.

In this article, the role of parasite shape in the alignment process at the RBC membrane is studied by mesoscopic computer simulations. In particular, we show that basic dynamical properties, such as parasite mobility, RBC membrane deformation, and the number of adhesion bonds, are significantly affected by different parasite shapes. In turn, these are tightly coupled to parasite alignment characteristics that determine its alignment success. In general, parasite shapes with large aspect ratios (e.g., oblate and long prolate ellipsoid) are disadvantageous for alignment, as these shapes result in a significant reduction of parasite mobility at the membrane. A spherical parasite is more mobile than an egg-like merozoite, which is advantageous in cases with low adhesion interactions or increased membrane stiffness. However, the spherical shape is disadvantageous for strong adhesion interactions, when parasite mobility is suppressed, as parasite alignment by membrane wrapping is often unsuccessful because the apex may not be within the adhesion area. As a result, the egg-like shape exhibits an alignment performance that is generally not worse than of other studied shapes, but more robust for disparate conditions in parasite adhesion strength and RBC membrane deformability. 
(a)

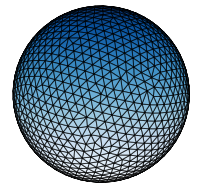

Sphere (SP)

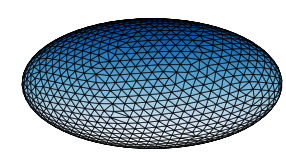

Short-elliposid (SE)

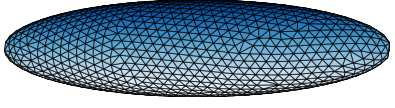

Long-elliposid (LE)

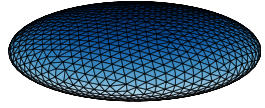

Oblate $(\mathrm{OB})$ (b)
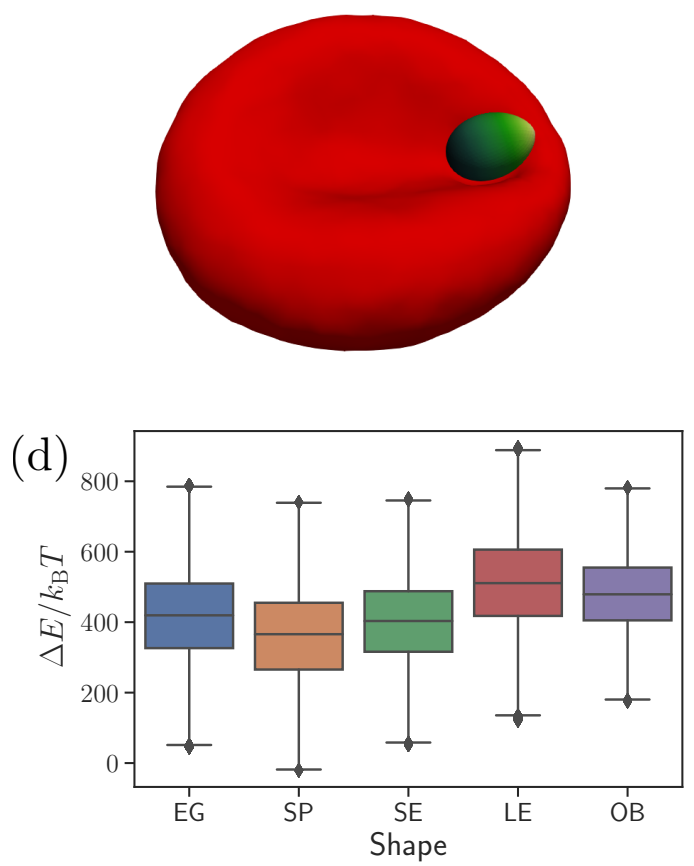
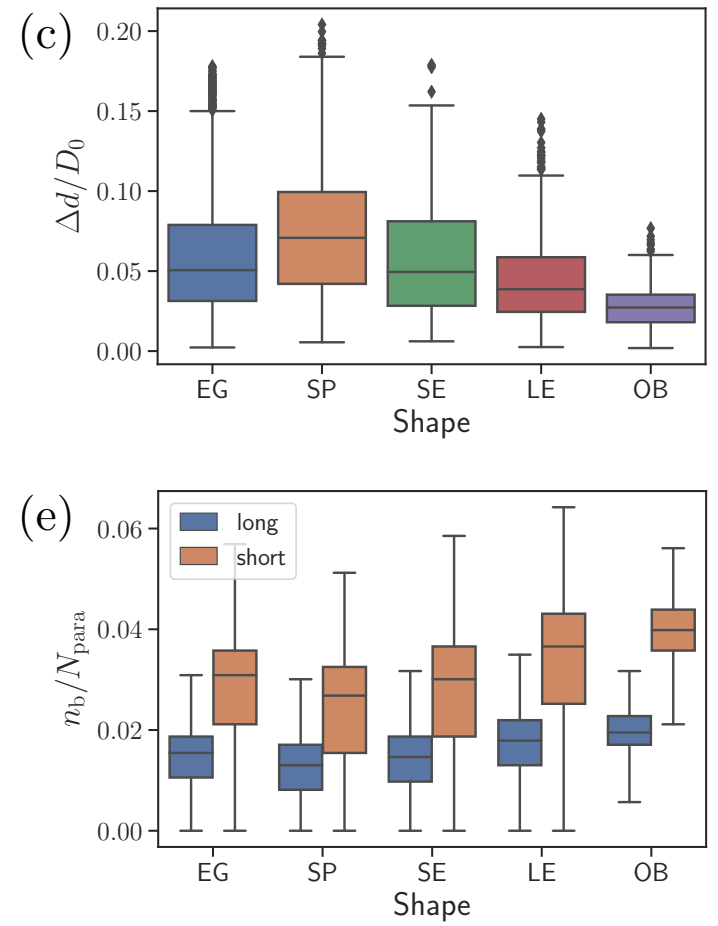

Figure 1. Different parasite shapes and their dynamic properties. (a) Triangulated surfaces of different parasite shapes including a sphere (SP), a short ellipsoid (SE), a long ellipsoid (LE), and an oblate ellipsoid (OB). (b) A snapshot from simulations showing an egg-like parasite interacting with the RBC membrane, see also Video 1. A bright yellow color indicates the apical complex and a dark green color represents the parasite's back. The egg-like (EG) shape is a typical shape of merozoites. (c-e) Different dynamical characteristics for various parasite shapes. (c) Fixed-time displacement $\Delta d$ of the parasite normalized by an effective RBC diameter $D_{0}=\sqrt{A_{0} / \pi}$ where $A_{0}$ is the membrane area. (d) Change in total membrane energy $\Delta E$ due to deformation induced by the parasite. (e) Number of short and long bonds $n_{\mathrm{b}}$.

\section{Results}

To investigate the role of parasite's shape in the alignment process, five different shapes with varying aspect ratios are chosen: $(i)$ an egg-like (EG) shape that is the typical shape of Plasmodium falciparum merozoite, (ii) a sphere (SP), (iii) a short ellipsoid (SE) whose dimensions are similar to the egg-like shape, $(i v)$ a long ellipsoid (LE), and $(v)$ an oblate (OB) shape, see Fig. 1(a). The corresponding maximum and minimum dimensions for these shapes are $r_{\max }=1.5 \mu \mathrm{m} \& r_{\min }=1.08 \mu \mathrm{m}$ for EG, $r_{\max }=r_{\min }=1.2 \mu \mathrm{m}$ for SP, $r_{\max }=1.6 \mu \mathrm{m} \& r_{\min }=1.02 \mu \mathrm{m}$ for SE, $r_{\max }=2.4 \mu \mathrm{m} \& r_{\min }=0.76 \mu \mathrm{m}$ for $\mathrm{LE}$, and $r_{\max }=1.5 \mu \mathrm{m} \& r_{\min }=0.64 \mu \mathrm{m}$ for $\mathrm{OB}$. All shapes are selected such that they have approximately the same surface area and the same number of vertices or equivalently the same density of adhesion receptors. The fraction of receptors that can form long bonds is kept at 0.4 similarly to our previous study (Hillringhaus et al., 2020), while the fraction of receptors for short bonds is equal to 0.6. Other parasite-RBC interaction parameters are calibrated through the displacement of an egg-shaped parasite at RBC membrane [see Fig. 1(b) and Video 1] against available experimental data (Weiss et al., 2015). Note that the translational displacement of the parasite results from its stochastic rolling-like motion. The set of calibrated parameters will be 


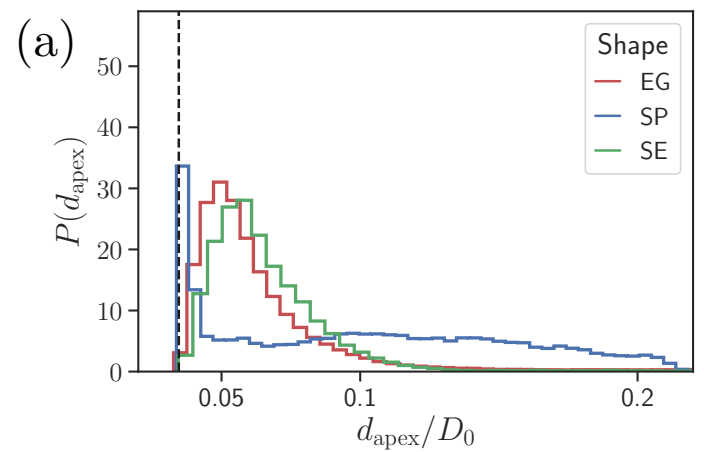

(c)

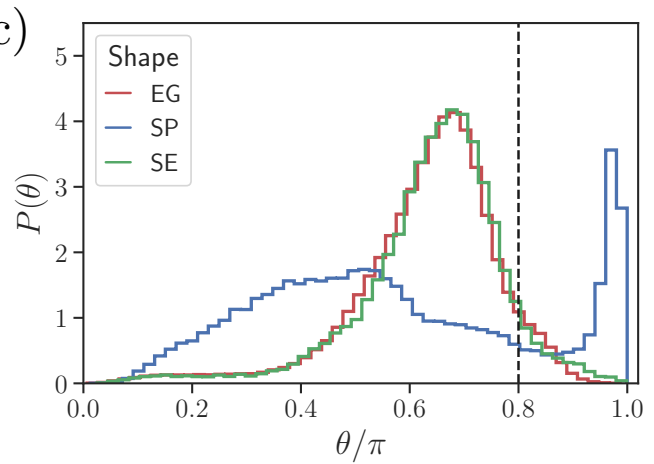

referred to as the reference parameter set, see Hillringhaus et al. (2020) for more details. All other parameters including bond rates are kept same unless stated otherwise.
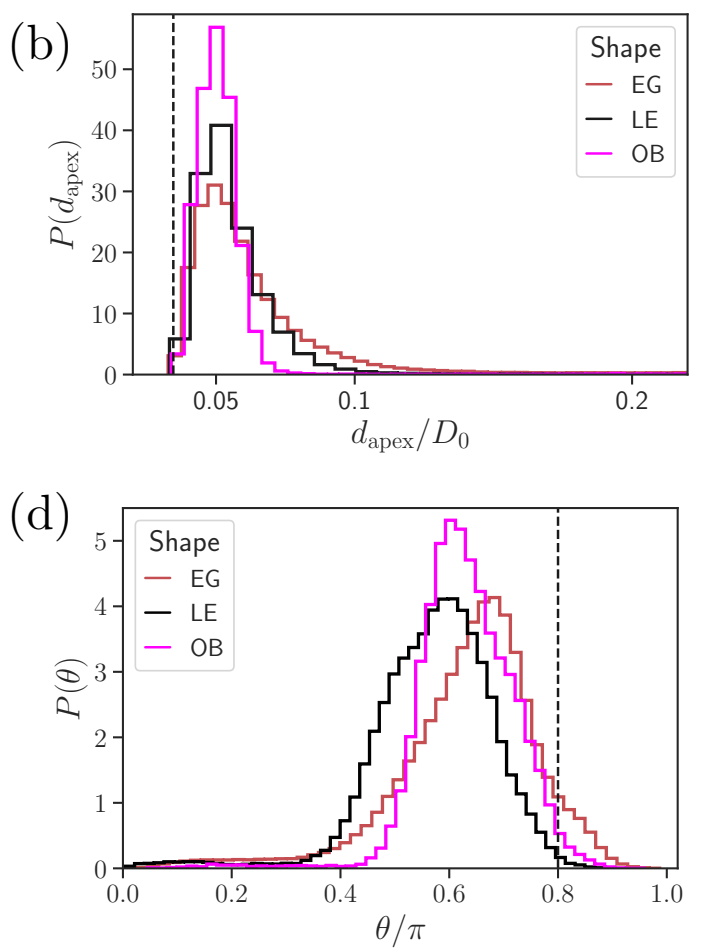

Figure 2. Parasite alignment characteristics. (a) \& (b) Apex distance $d_{\text {apex }}$ distributions and (c) \& (d) alignment angle $\theta$ distributions for all shapes. In all plots, the alignment criteria from Eq. (2) are shown by the dashed lines. For a better readability, the distributions for SP and SE shapes in (a) \& (c) are plotted separately from the distributions for LE and OB shapes in (b) \& (d) along with egg-like shape (EG) distributions in all plots.

\section{Dynamical properties of different shapes}

Figure $1(c-e)$ presents basic dynamical measures of merozoites with different shapes. These include [Fig. 1(c)] fixed-time displacements $\Delta d$ traveled by the parasite over fixed time intervals of $\Delta t=1 \mathrm{~s}$ and normalized by an effective RBC diameter $D_{0}=\sqrt{A / \pi}$ ( $A$ is the membrane area), [Fig. 1(d)] change in membrane total energy $\Delta E / k_{\mathrm{B}} T$ due to deformation induced by parasite adhesion, and [Fig. 1 (e)] the number of bonds $n_{\mathrm{b}} / N_{\text {para }}$. The spherical shape is most mobile (i.e., has the largest $\Delta d / D_{0}$, see Video 2), while the oblate shape is slowest with the lowest $\Delta d$. Intuitively, shape with a larger aspect ratio should form a larger adhesion area, and thus be less dynamic or mobile. This is in agreement with our results in Fig. 1(e), where the SP (OB) shape has the smallest (largest) number of bonds, which is directly proportional to the adhesion area. Note that the egg-like and short-ellipsoid shapes show very similar dynamic characteristics, as these shapes are very close to each other. Fixed-time displacement of the long ellipsoid (see Video 2 ) has values between those for the SE and OB shapes.

The RBC deformation energy $E / k_{\mathrm{B}} T$ can generally be expected to be proportional to the adhesion area or the number of formed bonds. This is true for the spherical shape that induces the lowest deformation energy in Fig. 1(d). Furthermore, both oblate and long ellipsoid shapes result in a large deformation energy. However, the LE shape has a slightly larger value of $\Delta E$ than the OB shape, even though the oblate shape has a larger adhesion area. This can be rationalized by the fact that the adhesion of oblate shape to RBC membrane induces a lower deformation than the LE shape as the OB shape has a lower curvature at its flat side. Note that the RBC deformation energy displays opposite trends in its shape dependence than the parasite mobility or fixed-time 
displacement in Fig. 1(c). As a result, parasite shapes with a large asphericity are less dynamic at the RBC surface, while the EG, SP, and SE shapes show comparable dynamical characteristics.

\section{Parasite alignment characteristics}

To characterize parasite alignment, we introduce two quantities: (i) apex distance $d_{\text {apex }}$ and (ii) alignment angle $\theta$ (Hillringhaus et al., 2020) given by

$$
d_{\text {apex }}=\min _{i}\left(\left|\mathbf{r}_{\text {apex }}-\mathbf{r}_{i}\right|\right), \quad \theta=\arccos \left(\mathbf{n} \cdot \mathbf{n}^{\text {face }}\right),
$$

where $\mathbf{r}_{\text {apex }}$ is the apex position, $\mathbf{r}_{i}$ is the position of vertex $i$ at the membrane, $\mathbf{n}$ is the parasite's directional vector pointing from its back to the apex, and $\mathbf{n}^{\text {face }}$ is the normal vector of a RBC membrane triangular face whose center of mass is closest to the parasite's apex. The directional vector is defined for all shapes by selecting two opposite vertices along the shape axis, which represent the apex and the back. Following our previous work (Hillringhaus et al., 2020), a successful parasite alignment can be characterized by the criteria

$$
d_{\text {apex }} \leq 2^{1 / 6} \sigma+r_{\text {junc }} \quad \& \quad \theta \geq 0.8 \pi,
$$

where $r_{\text {junc }}=10 \mathrm{~nm}$ defines the junctional interaction range of the parasite's apex (Bannister et al., 1986b).

Figure 2 presents apex-distance and alignment-angle distributions for different parasite shapes, where the alignment criteria from Eq. (2) are indicated by the dashed lines. Even though alignment of the SE shape is similar to the EG shape, it is slightly worse for the SE shape as the $d_{\text {apex }}$ distribution in Fig. 2(a) is shifted further away from the alignment criterion for $d_{\text {apex }}$ than that for the EG shape. Furthermore, the egg-like shape aligns better than the long-ellipsoid and oblate shapes, which is supported by the alignment-angle distributions in Fig. 2(d). Clearly, parasite alignment characteristics for the spherical shape are qualitatively different from all other (ellipsoid-like) shapes. From the first glance, $d_{\text {apex }}$ and $\theta$ distributions in Fig. 2(a) \& (c) seem to suggest that the SP shape might be best for parasite alignment. However, alignment performance of the spherical parasite is more subtle than indicated by these distributions and will be discussed further.
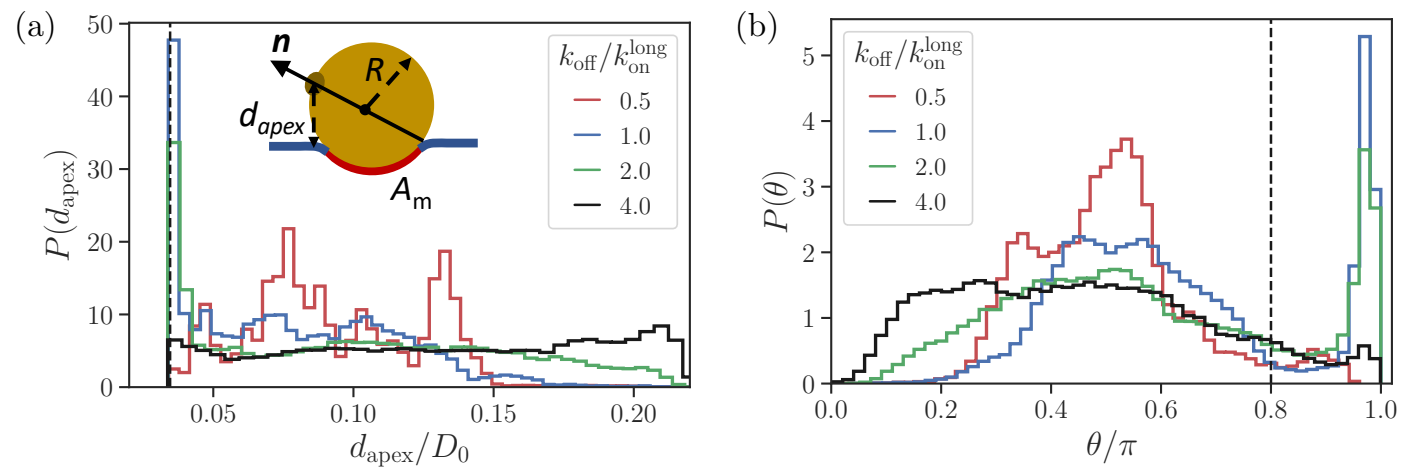

Figure 3. Alignment of a spherical parasite. (a) Apex-distance and (b) alignment-angle distributions of the SP shape for different bond off-rates. The alignment criteria from Eq. 2 are shown by the dashed lines. The inset in (a) sketches a sphere of radius $R$ partially wrapped by membrane area $A_{\mathrm{m}}$, where n denotes directional vector of the parasite. Several peaks in $P\left(d_{\text {apex }}\right)$ for $k_{\text {off }} / k_{\text {on }}^{\text {long }}=0.5$ are due to limited statistics, since for the strongest adhesion, the parasite mobility is very low and the simulations are too short to fully capture this distribution.

\section{Alignment of a spherical parasite}

Figure 3 shows apex-distance and alignment-angle distributions of the spherical parasite for different values of the off-rate $k_{\text {off }}$. Both $d_{\text {apex }}$ and $\theta$ distributions generally display a sharp peak near 
the alignment criteria and a long tail with a wider distribution for non-aligned parasite orientations. Thus, the sharp peak represents parasite orientations when its apex is within the membraneparasite contact area $A_{\mathrm{m}}$, as schematically illustrated in the inset of Fig. 3(a). Correspondingly, the long tail characterizes orientations when the parasite's apex is not within the adhesion area $A_{\mathrm{m}}$.

At large values of the off-rate (e.g., $k_{\text {off }} / k_{\text {on }}^{\text {long }}=4.0$ ), the spherical parasite is very mobile at the membrane surface (i.e., has a large effective rotational diffusion) and induces nearly no membrane deformations. In such cases, there is no significant wrapping of the parasite by the membrane, resulting in wide $d_{\text {apex }}$ and $\theta$ distributions in Fig. 3 . As $k_{\text {off }}$ is decreased, the merozoite becomes partially wrapped by the membrane, leading to the development of the sharp peak in both distributions. At the smallest off-rate of $k_{\text {off }} / k_{\text {on }}^{\text {long }}=0.5$, the alignment properties in Fig. 3 seem to be qualitatively different from those for larger off-rates. Note that for small off-rates, the parasite forms a large number of bonds with the membrane, resulting essentially in its arrest with nearly zero rotational diffusion. Therefore, these simulations are too short to fully capture $d_{\text {apex }}$ and $\theta$ distributions, which are also expected to have the sharp peak characterized by the wrapped area $A_{\mathrm{m}}$.

To rationalize apex-distance and alignment-angle distributions for a spherical parasite, we use a simple model of a sphere with radius $R$ partially wrapped by the membrane, as illustrated in the inset of Fig. 3(a). Since the adhered parasite is mobile, the parasite's directional vector can point toward any possible direction when sampled over times longer than a characteristic time of parasite rotational motion. Therefore, the probability of alignment can be approximated as $A_{\mathrm{m}} / A_{\mathrm{s}}$, where $A_{\mathrm{m}}$ and $A_{\mathrm{s}}$ are the adhesion area and the total surface area of a sphere, respectively. As a result, the sharp peak in $d_{\text {apex }}$ and $\theta$ distributions near the alignment criteria must increase with an increase in adhesion strength or a decrease in $k_{\text {off }}$.

When the parasite is not aligned, the apex distance can be approximated by a height of the parasite's apex with respect to the flat part of the membrane, see the inset of Fig. 3(a). Note that this assumption becomes strictly valid for a flat membrane without wrapping or a weak adhesion of the merozoite. Then, the apex probability is characterized by the area of thin rings at the sphere surface as

$$
P\left(d_{\text {apex }}\right) \Delta h=\frac{A_{\Delta h}}{A_{\mathrm{s}}}=\frac{2 \pi R \Delta h}{A_{\mathrm{s}}}=\frac{1}{2 R} \Delta h,
$$

where $\Delta h$ is an infinitesimally small interval around $d_{\text {apex }}$. Therefore, $P\left(d_{\text {apex }}\right)=1 /(2 R)$ is independent of the apex distance, which is consistent with nearly flat $d_{\text {apex }}$ distributions for $k_{\text {off }} / k_{\text {on }}^{\text {long }} \geq 2.0$ in Fig. 3(a). Similarly, the angle probability $P(\theta)$ can be approximated using ring areas $A_{\Delta \theta}=2 \pi R^{2}(\cos (\theta)-\cos (\theta+\Delta \theta)) \approx 2 \pi R^{2} \sin (\theta) \Delta \theta$, resulting in

$$
P(\theta) \approx \frac{\sin (\theta)}{2}
$$

for not aligned parasite orientations. This approximation is consistent with the data in Fig. 3(b). In summary, such unique distributions of alignment properties for the SP shape are possible due to the spherical symmetry. For non-spherical parasite shapes, the sharp peak disappears because parasite adhesion to the membrane favors a specific apex orientation.

\section{Effect of adhesion strength on parasite alignment time}

Figure 4(a) and (b) show apex-distance and alignment-angle properties for different parasite shapes and various off-rates which is used to control the strength of merozoite adhesion to the membrane. The apex distance decreases when the off-rate is decreased or the strength of adhesion is increased. Similarly, the alignment angle increases toward the alignment criterion in Eq. (2), as the adhesion strength is increased. For all non-spherical shape cases, successful alignment is generally achieved at low enough $k_{\text {off }}$ values, which imply strong membrane deformations and a significant wrapping of the parasite by the membrane. This is consistent with deformation energies shown in Fig. 4(c), which significantly increase with decreasing $k_{\text {off }}$. The main difference for the spherical parasite is that the best alignment is achieved for intermediate values of off-rates (e.g., $k_{\text {off }} / k_{\text {on }}^{\text {long }} \approx 1.0$ ). As 

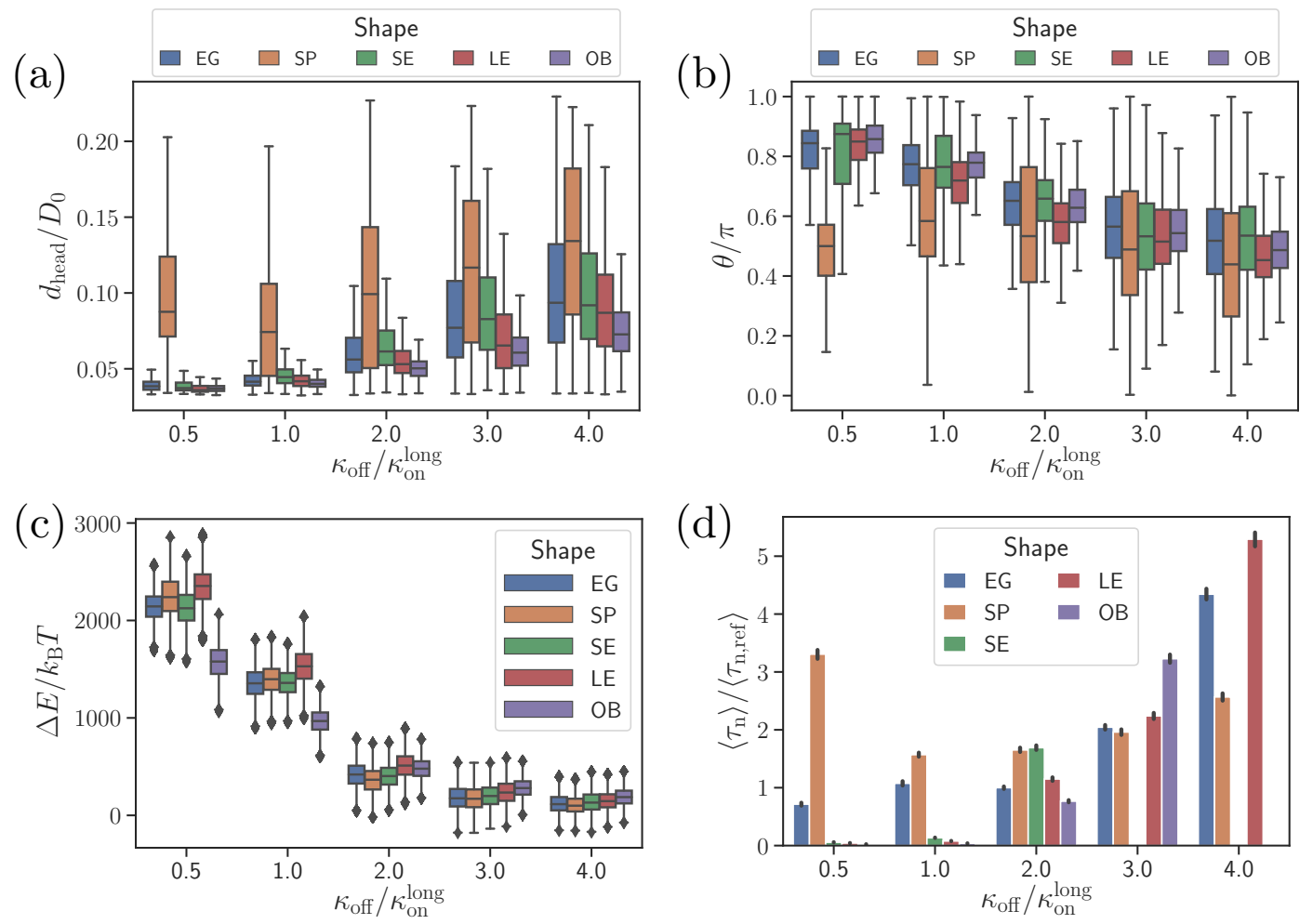

Figure 4. Effect of parasite adhesion strength of the alignment time. (a) Apex distance $d_{\text {apex }}$ (b) alignment angle $\theta$, (c) total deformation energy $\Delta E$, and (d) alignment time $\tau_{\mathrm{n}}$ for different parasite shapes and bond off-rates that determine the adhesion strength. Several missing bars in the plot of alignment times for $k_{\text {off }} / k_{\text {on }}^{\text {long }}>2$ indicate that $\tau_{\mathrm{n}}$ is much larger than 26 seconds which is the maximum time of all simulation trajectories.

mentioned before, small values of $k_{\text {off }}$ significantly suppress parasite mobility, which is required for successful alignment of the spherical parasite because its apex may not be immediately within the parasite-membrane contact area after initial adhesion.

To compute alignment times, we employ Monte Carlo simulations based on $\left(d_{\text {apex }}, \theta\right)$ probability maps constructed from approximately 10 independent direct simulation trajectories for each parameter set (Hillringhaus et al., 2020). Figure 4 (d) shows alignment times $\tau_{\mathrm{n}}$ for different parasite shapes and off-rates, where all times are normalized by the alignment time of an egg-like shape for the reference parameter set (Hillringhaus et al., 2020). In some cases, the bars are missing in the plot, indicating that the alignment has not occurred in direct simulations whose maximum time length is about 26 seconds. Alignment times of the spherical parasite are very long at small off-rates and become comparable with those of the egg-like shape at intermediate and high values of $k_{\text {off }}$. The SE, LE, and OB shapes generally align very fast at small off-rates, but often do not align at all when adhesion becomes weak. This means that these spheroidal shapes require substantial membrane deformation for a successful alignment.

\section{Alignment at a rigid RBC}

To understand the importance of RBC deformability in the alignment process for different parasite shapes, we have simulated parasite alignment at a rigid RBC. Figure 5(a) and (b) presents fixed-time displacement and alignment time for different parasite shapes and two off-rates. Generally, a small fixed-time displacement (or low mobility at the membrane) results in a long alignment time and vise versa. Both long-ellipsoid and oblate shapes do not align or have a very long alignment time at a rigid membrane, as they require considerable amount of membrane deformation for the alignment. 

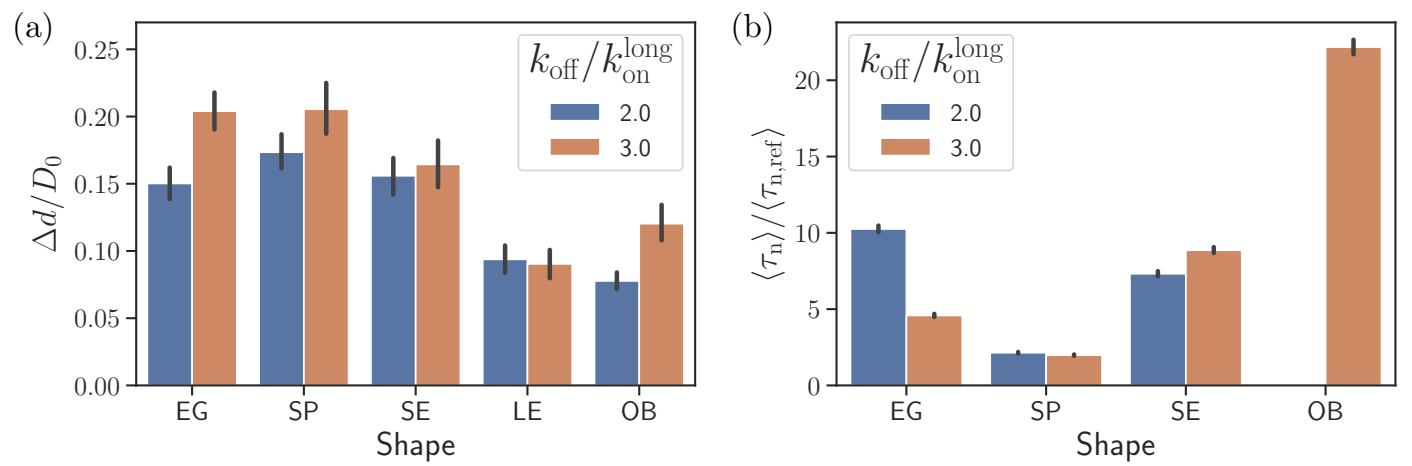

Figure 5. Alignment at a rigid membrane. (a) Fixed-time displacement $\Delta d / D_{0}$ and (b) alignment time $\left\langle\tau_{\mathrm{n}}\right\rangle /\left\langle\tau_{\mathrm{n}, \text { ref }}\right\rangle$ for different parasite shapes and two values of off-rates $k_{\text {off }} / k_{\text {on }}^{\text {long }}$. Note that the data for long ellipsoids is omitted as they never become aligned during direct simulations indicating that their alignment time is much larger than the total simulation time.

Both egg-like shape and short ellipsoid exhibit similar fixed-time displacements and alignment times. However, for the egg-like shape, an increase in off-rate (i.e., more mobility) improves the alignment whereas for the short ellipsoid an opposite trend is observed. The spherical parasite shows the fastest alignment in comparison to the egg-like and short ellipsoid shapes due to its increased mobility. Thus, at a rigid RBC, the spherical shape shows best alignment properties, at least for intermediate off-rate values.

\section{Discussion and conclusions}

We have investigated the importance of merozoite shape for its alignment at the RBC membrane which is a prerequisite for the invasion process. This study is a continuation of our previous work (Hillringhaus et alo, 2020) where the alignment of an egg-like parasite, a natural shape of merozoite, was investigated. Motivated by experimental observations (Bannister et al., 1986a), adhesion between the RBC membrane and the parasite is implemented by discrete bonds of two different types, with long and short interaction ranges. The density of both long and short bonds, their kinetic rates and extensional rigidities are calibrated through fixed-time displacement of an egg-like shaped parasite against available experimental data (Weiss et al., 2015). Alignment times from two independent experiments are found to be $16 \mathrm{~s}$ (Weiss et al., 2015) and $7-44 \mathrm{~s}$ (Yahata et al., 2012), respectively. For the egg-like shape, an average alignment time of $\simeq 10 \mathrm{~s}$ was obtained in our simulations (Hillringhaus et al., 2020). To study the effect of parasite shape on alignment, five different parasite shapes, including the original egg-like (EG) shape, short ellipsoid (SE), sphere (SP), long ellipsoid (LE) and oblate (OB) shapes, were considered. In general, some parasite shapes are advantageous when the binding kinetics are slow or large RBC membrane deformations take place, while other shapes are advantageous in case of fast binding kinetics or small membrane deformations.

One of our key results is that the spherical parasite exhibits apex-distance and alignmentangle distributions different from those for non-spherical shapes. Distributions of alignment characteristics for the SP shape generally have a sharp peak near the alignment criteria representing parasite orientations with its apex within the adhesion area. At small off-rates or for strong adhesion interactions, there is a considerable parasite wrapping by the membrane and parasite mobility is suppressed. In this case, a successful alignment of the spherical parasite occurs only if the apex ends up directly within the wrapped part of the membrane. This means that the SP shape exhibits "all or nothing" alignment behavior at small off-rates. In contrast, the egg-like shape adheres with its side to the membrane, and is able to establish a direct membrane-apex contact due to significant parasite wrapping. On the other hand, the spherical shape performs better than the EG shape at large values of off-rates when membrane deformation is almost negligible. The spherical symmetry 

of the SP shape results in its faster mobility in comparison with the egg-like shape. Furthermore, a fluctuation of the EG parasite toward successful alignment due to adhesive dynamics is associated with a larger energetic barrier in comparison to the SP shape for which all directions of motion are statistically equivalent. This is also the main reason why the spherical shape leads to the fastest alignment at a rigid membrane in comparison to all other shapes. Interestingly, even though most types of Plasmodium merozoites have an egg-like shape, Plasmodium yoelii transforms into a spherical shape from an egg-like shape after the egress from an infected RBC (Yahata et al., 2012).

Short ellipsoid geometrically resembles the EG shape except that the egg-like shape has asymmetric ends along the cylindrical axis. Therefore, the SE shape shows alignment characteristics that are closest to the EG shape. However, the alignment of the SE shape is slightly worse than that of the egg-like parasite, as the alignment-angle distribution for short ellipsoid is shifted further away from the alignment criterion in comparison to the egg-like shape. This is due to the asymmetry of EG shape along the cylindrical axis, which favors an adhesion orientation tilted toward the apex. At low enough bond off-rates or strong adhesion interactions, alignment of the SE shape is faster than the egg-like shape, as the wrapping of more curved apex region of the EG parasite is slightly less energetically favorable than that of a symmetric SE shape. Furthermore, alignment of oblate and long-ellipsoid shapes proceeds only through significant wrapping of the parasite by the membrane, which occurs only at low off-rates or for strong adhesion interactions. This is also evident from simulations at a rigid $R B C$, where alignment times of the $L E$ and $O B$ shapes are either very long or no alignment potentially occurs. Despite the fact that the egg-like shape has some advantages over the investigated spheroidal shapes, it is not clear whether this asymmetry exists simply due to the internal parasite structure (e.g., placement of essential organelles of different sizes) or has some functional importance.

Finally, apical alignment at the RBC membrane is followed by parasite invasion, which requires the formation of a tight junction. During invasion, the tight junction is formed at the apical end and moves toward the back of the parasite with an aid of actomyosin machinery (Keeley and Soldati, 2004; Robert-Paganin et al., 2019; Cowman and Crabb, 2006). Even though the invasion includes mainy mechano-chemical processes, parasite shape must play an important role, as it significantly affects the energy required to deform RBC membrane. For instance, particles with a larger aspect ratio such as oblate and long ellipsoids require a larger energy for complete wrapping (or uptake) (Bahrami et al., 2014; Dasgupta et al., 2014a, 2017). From a dynamical perspective, fluctuations that are important for particle uptake also depend on the geometry of a particle (Frey et al., 2019). Note that particle uptake studies are performed majorly for vesicles, while RBCs possess shear elasticity in addition to membrane bending rigidity. For instance, Hillringhaus et al. (2019) show that for small interaction strengths, bending energy has a dominant contribution to membrane deformation energy, while for strong interactions shear elastic energy exhibits a more dominant contribution. Different aspects related to the performance of various parasite shapes for RBC invasion clearly require further investigations.

\section{Methods}

Both RBC and parasite are modeled as triangulated surfaces. The RBC is equipped with elastic and bending energies, while the parasite is treated as a rigid body. Both the RBC and parasite are immersed in a fluid which is modeled by the dissipative particle dynamics (DPD) method (Hoogerbrugge and Koelman, 1992). For details about RBC and parasite models, we refer the reader to Hillringhaus et al. (2020). Different parasite shapes are represented by triangulated surfaces with the surface area and the number of vertices approximately equal to those of the egg-like shape. This ensures that the receptor density is constant for all shapes.

\section{RBC-parasite interactions}

Parasite interacts with the RBC membrane in two ways including excluded volume and adhesion interactions. The excluded-volume interaction is implemented through the Lennard-Jones potential 
given by

$$
U_{\text {rep }}(r)=4 \epsilon\left[\left(\frac{\sigma}{r}\right)^{12}-\left(\frac{\sigma}{r}\right)^{6}\right], \quad r \leq 2^{1 / 6} \sigma,
$$

\section{Video description}

3łbideo 1: Motion of an egg-shaped parasite at the membrane of a deformable RBC for the reference $334 \quad$ RBC-parasite interactions. $k_{\text {off }} / k_{\text {on }}^{\text {long }}=2$.

336ideo 2: Mobility of the LE and SP shaped parasites at the RBC membrane, which can be compared to 336

where $r$ is the distance between RBC and parasite vertices, $\sigma$ is the repulsive distance chosen to be $0.2 \mu \mathrm{m}$, and $\epsilon=1000 k_{\mathrm{B}} T$ is the strength of interaction.

Adhesion interactions are represented by a discrete receptor-ligand bond model. As in our previous work (Hillringhaus et al., 2020), two different types of adhesion bonds are used: (i) long bonds with an effective length of $\ell_{\text {eff }}^{\text {long }}=100 \mathrm{~nm}$ and $(i i)$ short bonds with an effective length of $\ell_{\mathrm{eff}}^{\text {short }}=20 \mathrm{~nm}$. The fraction of long bonds is set to $\rho=0.4$, while the fraction of short bonds then becomes $1-\rho=0.6$. Adhesion bonds between the RBC and the parasite form and dissociate with constant on-rates $k_{\mathrm{on}}^{\text {long }}$ and $k_{\mathrm{on}}^{\text {short }}$, and an off-rate $k_{\text {off }}$ which is the same for both bond types. Both long and short bonds are modeled by a harmonic potential as

$$
U_{\text {ad }}(\ell)=\frac{\lambda_{\text {type }}}{2}\left(\ell-\ell_{0}\right)^{2},
$$

where $\lambda_{\text {long }}$ and $\lambda_{\text {short }}$ are the extensional rigidities of long and short bonds, respectively. $\ell_{0}=2^{1 / 6} \sigma$ is the equilibrium bond length. Thus, long bonds are formed when the distance between parasite and membrane vertices is less than $\ell_{0}+\ell_{\mathrm{eff}}^{\text {long }}$ and short bonds can form when $\ell<\ell_{0}+\ell_{\mathrm{eff}}^{\text {short }}$.

\section{Simulation setup}

All simulations are carried out in a simulation domain of size $7.7 D_{0} \times 3.1 D_{0} \times 3.1 D_{0}$ with periodic boundary conditions in all directions, where $D_{0}=\sqrt{A_{0} / \pi}$ is the effective RBC diameter and $A_{0}$ is the membrane area. Receptors for both long and short bonds are chosen randomly over the parasite surface and this procedure is repeated for every realization to obtain a good averaging of physical quantities. In every simulation, the parasite is placed close to the RBC membrane in order to facilitate its initial attachment. The initial position of the parasite is with its back toward the membrane, so that its apex is directed away from the membrane. RBC bending rigidity is chosen to be $\kappa=3 \times 10^{-19} \mathrm{~J}$ and $A_{0}=133 \mu \mathrm{m}^{2}$ resulting in $D_{0}=6.5 \mu \mathrm{m}$. Fluid viscosity inside and outside the RBC is set to $\eta=1 \mathrm{mPa}$.s. To connect simulation and physical units, we use $D_{0}$ as a length scale, $k_{\mathrm{B}} T$ as an energy scale, and the RBC membrane relaxation time $\eta D_{0}^{3} / \kappa$ as a time scale. All simulations were performed on JURECA, a super-computer at Forschungszentrum Jülich Vülich Supercomputing Centre, 2018).

\section{Alignment times: Monte Carlo sampling}

A Monte Carlo sampling scheme is employed for measuring alignment times from probability maps of parasite alignment characteristics (apex distance $d_{\text {apex }}$ and alignment angle $\theta$ ), which are constructed from approximately 10 independent long simulations for each parameter set. Briefly, the Monte Carlo procedure is as follows. First, a state $(i, j)$ is randomly selected, which corresponds to specific $\left(d_{\text {apex }}^{i}, \theta_{j}\right)$ values in a probability map. Second, a transition to one of the four neighboring states with a probability of 0.25 is attempted, and it is accepted if $\zeta<P$ (new state) $/ P(i, j)$ where $\zeta$ is a uniform random number. This state transition is repeated until a state that meets the alignment criteria is reached. Then, the total alignment time is equal to the total number of Monte Carlo moves, see Hillringhaus et al. (2020) for more details. All alignment times are normalized by the corresponding time for the reference parameter set, which is obtained through the calibration of parasite speed (Hillringhaus et al., 2020) against available experimental data (Weiss et al., 2015). 


\section{Author Contributions}

A.K.D and S.H. performed all the simulations and analyzed the computational results; G.G. and

D.A.F. designed the research project; all authors interpreted the results and wrote the manuscript.

\section{Acknowledgments}

Sebastian Hillringhaus acknowledges support by the International Helmholtz Research School of Biophysics and Soft Matter (IHRS BioSoft). We gratefully acknowledge the computing time granted through JARA-HPC on the supercomputer JURECA (Jülich Supercomputing Centre, 2018) at Forschungszentrum Jülich.

\section{Competing interests}

The authors declare no competing interests.

\section{References}

Bahrami AH, Raatz M, Agudo-Canalejo J, Michel R, Curtis EM, Hall CK, Gradzielski M, Lipowsky R, WeikI TR. Wrapping of nanoparticles by membranes. Adv Colloid Interface Sci. 2014; 208:214-224.

Bannister LH, Mitchell GH, Butcher GA, Dennis ED. Lamellar membranes associated with rhoptries in erythrocytic merozoites of Plasmodium knowlesi: a clue to the mechanism of invasion. Parasitology. 1986; 92:291-303.

Bannister LH, Mitchell GH, Butcher GA, Dennis ED, Cohen S. Structure and development of the surface coat of erythrocytic merozoites of Plasmodium knowlesi. Cell Tissue Res. 1986; 245:281-290.

Beeson JG, Drew DR, Boyle MJ, Feng G, Fowkes FJI, Richards JS. Merozoite surface proteins in red blood cell invasion, immunity and vaccines against malaria. FEMS Microbiol Rev. 2016; 40:343-372.

Cowman AF, Berry D, Baum J. The cellular and molecular basis for malaria parasite invasion of the human red blood cell. J Cell Biol. 2012; 198:961-971.

Cowman AF, Crabb BS. Invasion of red blood cells by malaria parasites. Cell. 2006; 124:755-766.

Crick AJ, Theron M, Tiffert T, Lew VL, Cicuta P, Rayner JC. Quantitation of malaria parasite-erythrocyte cell-cell interactions using optical tweezers. Biophys J. 2014; 107:846-853.

Crick AJ, Tiffert T, Shah SM, Kotar J, Lew VL, Cicuta P. An automated live imaging platform for studying merozoite egress-invasion in malaria cultures. Biophys J. 2013; 104:997-1005.

Dasgupta S, Auth T, Gompper G. Shape and orientation matter for the cellular uptake of nonspherical particles. Nano Lett. 2014; 14:687-693.

Dasgupta S, Auth T, Gompper G. Nano- and microparticles at biological and fluid interfaces. J Phys Condens Matter. 2017; 29:373003.

Dasgupta S, Auth T, Gov N, Satchwell TJ, Hanssen E, Zuccala ES, Riglar DT, Toye AM, Betz T, Baum J, Gompper G. Membrane-wrapping contributions to malaria parasite invasion of the human erythrocyte. Biophys J. 2014; 107:43-54.

Dvorak JA, Miller LH, Whitehouse WC, Shiroishi T. Invasion of erythrocytes by malaria merozoites. Science. $1975 ; 187: 748-750$.

Frey F, Ziebert F, Schwarz US. Stochastic dynamics of nanoparticle and virus uptake. Phys Rev Lett. 2019; 122:088102.

Gilson PR, Crabb BS. Morphology and kinetics of the three distinct phases of red blood cell invasion by Plasmodium falciparum merozoites. Int J Parasitol. 2009; 39:91-96.

Gilson PR, Nebl T, Vukcevic D, Moritz RL, Sargeant T, Speed TP, Schofield L, Crabb BS. Identification and stoichiometry of glycosylphosphatidylinositol-anchored membrane proteins of the human malaria parasite Plasmodium falciparum. Mol Cell Proteomics. 2006; 5:1286-1299. 
Glushakova S, Yin D, Li T, Zimmerberg J. Membrane transformation during malaria parasite release from human red blood cells. Curr Biol. 2005; 15:1645-1650.

Hillringhaus S, Dasanna AK, Gompper G, Fedosov DA. Importance of erythrocyte deformability for the alignment of malaria parasite upon invasion. Biophys J. 2019; 117:1202-1214.

Hillringhaus S, Dasanna AK, Gompper G, Fedosov DA. Stochastic bond dynamics facilitates alignment of malaria parasite at erythrocyte membrane upon invasion. eLife. 2020; 9:e56500.

Hoogerbrugge PJ, Koelman JMVA. Simulating microscopic hydrodynamic phenomena with dissipative particle dynamics. Europhys Lett. 1992; 19:155-160.

Jülich Supercomputing Centre. JURECA: Modular supercomputer at Jülich Supercomputing Centre. J LargeScale Res Facil. 2018; 4:A132.

Keeley A, Soldati D. The glideosome: a molecular machine powering motility and host-cell invasion by Apicomplexa. Trends Cell Biol. 2004; 14:528-532.

Koch M, Baum J. The mechanics of malaria parasite invasion of the human erythrocyte - towards a reassessment of the host cell contribution. Cell Microbiol. 2016; 18:319-329.

Miller LH, Baruch DI, Marsh K, Doumbo OK. The pathogenic basis of malaria. Nature. 2002; 415:673-679.

Robert-Paganin J, Robblee JP, Auguin D, Blake TCA, Bookwalter CS, Krementsova EB, Moussaoui D, Previs MJ, Jousset G, Baum J, Trybus KM, Houdusse A. Plasmodium myosin A drives parasite invasion by an atypical force generating mechanism. Nat Comm. 2019; 10:3286.

Weiss GE, Gilson PR, Taechalertpaisarn T, Tham WH, de Jong NWM, Harvey KL, Fowkes FJI, Barlow PN, Rayner JC, Wright GJ, Cowman AF, Crabb BS. Revealing the sequence and resulting cellular morphology of receptor-ligand interactions during Plasmodium falciparum invasion of erythrocytes. PLoS Pathog. 2015; 11:e1004670.

White NJ, Pukrittayakamee S, Hien TT, Faiz MA, Mokuolu OA, Dondorp AM. Malaria. Lancet. 2014; 383:723-735.

Yahata K, Treeck M, Culleton R, Gilberger TW, Kaneko O. Time-lapse imaging of red blood cell invasion by the rodent malaria parasite Plasmodium yoelii. PLoS ONE. 2012; 7:e50780. 